\title{
Long-lived Hot Carriers in III-V Nanowires
}

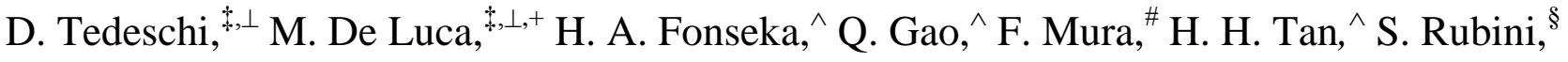
F. Martelli, ${ }^{\wedge}$ C. Jagadish, ${ }^{\wedge}$ M. Capizzi, ${ }^{\ddagger}$ and A. Polimeni ${ }^{\ddagger, *}$

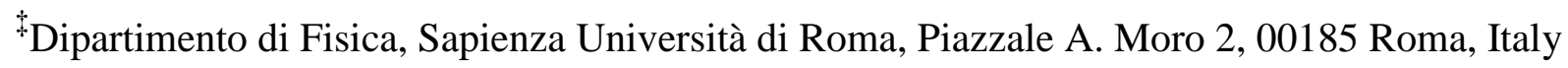

${ }^{\wedge}$ Department of Electronic Materials Engineering, Research School of Physics and Engineering, The Australian National University, Canberra, ACT 2601, Australia

" Dipartimento di Chimica and Sapienza Nanoscience \& Nanotechnology Lab (SNN-LAB), Sapienza Università di Roma, Piazzale A. Moro 2, 00185 Roma, Italy

${ }^{\S}$ Istituto Officina dei Materiali CNR, Basovizza SS 14 km 163.5, 34149 Trieste, Italy

${ }^{\Delta}$ Istituto per la Microelettronica e i Microsistemi CNR, Via del fosso del cavaliere 100, 00133 Roma, Italy

${ }^{\perp}$ These authors contributed equally to this work.

*E-mail addresses: antonio.polimeni@ roma1.infn.it

+Present address: Department of Physics, University of Basel, Klingelbergstrasse 82, 4056 Basel, Switzerland 


\section{S1) Growth details of the samples}

\section{InP NWs}

All InP nanowires (NWs) were grown in a horizontal flow low-pressure (100 mbar) metalorganic vapor-phase epitaxy (MOVPE) system (Aixtron 200/4).

a) InP WZ NWs; Selective Area Epitaxy (SAE) The array of wurtzite (WZ) InP NWs shown in Figure 1 (a) in the main text was grown by selective-area MOVPE or SAE on a (111)A InP substrate. Before NW growth, a 30 nm-thick $\mathrm{SiO}_{2}$ layer was deposited on an $\mathrm{InP}$ substrate and patterned by electron beam lithography to create an array of holes. The pattern was then transferred to the InP substrate by etching the $\mathrm{SiO}_{2}$ mask using a buffered hydrogen fluoride solution. We designed four different patterns to grow NWs with different diameters. After etching, the diameters of the holes were $45 \mathrm{~nm}, 120 \mathrm{~nm}, 310 \mathrm{~nm}$, and $650 \mathrm{~nm}$, and the pitch was 500, 200, 600, and 1000 $\mathrm{nm}$, respectively. The patterned substrates were then placed in the MOVPE system and annealed at $750{ }^{\circ} \mathrm{C}$ for 10 minutes under a protective flow. Finally, NWs were grown at $730{ }^{\circ} \mathrm{C}$ for 20 minutes with trimethylindium (TMIn) and phosphine $\mathrm{PH}_{3}$ at flow rates of $6.1 \times 10^{-6}$ and $4.9 \times 10^{-4} \mathrm{~mol} / \mathrm{min}$ (V/III ratio=80), respectively.

b) InP WZ NWs; Vapor Liquid Solid (VLS) The ensemble of WZ NWs displayed in Figure 1 (b) in the main text was grown by Au-seeded MOVPE on a semi-insulating (111)B InP substrate. Gold nanoparticles with diameter of $30 \mathrm{~nm}$ that acted as seeds for the VLS growth of the NWs were dispersed on the substrate. The samples were annealed under $\mathrm{PH}_{3}$ flow at $600{ }^{\circ} \mathrm{C}$ for 10 min before carrying out growth at the temperature of $480{ }^{\circ} \mathrm{C}$ and a V/III ratio of 350 for 20 min using TMIn and $\mathrm{PH}_{3}$ as precursors. The TMIn and $\mathrm{PH}_{3}$ precursor flows were $1.2 \times 10^{-5}$ and $4.2 \times 10^{-3} \mathrm{~mol} / \mathrm{min}$, respectively. We also investigated a sample grown with the same parameters, except for a V/III 
ratio equal to 110 . The two samples feature very similar morphological, structural, and optical properties.

c) InP WZ/ZB NWs; VLS The WZ/zincblende (ZB) NWs shown in Figure 1 (c) in the main text were grown on a semi-insulating InP (100) substrate using $30 \mathrm{~nm}$ Au particles as seeds for the VLS process. The substrate was annealed at $390{ }^{\circ} \mathrm{C}$ under $\mathrm{PH}_{3}$ flow for 10 min before ramping the temperature to a growth temperature of $475{ }^{\circ} \mathrm{C}$. Growth was initiated with TMIn and $\mathrm{PH}_{3}$ flows corresponding to a V/III ratio of 350 to ensure a high yield of vertical [100] segments. After 15 min, the V/III ratio was reduced to 220 in order to minimize lateral growth. Growth continued for a further 165 min before switching off TMIn and cooling down under $\mathrm{PH}_{3}$ flow. After growing for a length of $\sim 1.4 \mu \mathrm{m}$ (approximately $90 \mathrm{~min}$ of growth), all [100] wires kinked to a $<111>\mathrm{B}$ orientation by forming an inclined twin. The spontaneous change of growth direction took place without any change in the growth parameters. The [100] oriented segment before the inclined twin is of $\mathrm{ZB}$ phase and the segment grown after establishing the $<111>$ growth direction is of WZ phase with very few stacking faults.

d) InP ZB NWs; VLS The ZB NWs shown in Figure 1 (d) in the main text were grown by Auseeded MOVPE on a semi-insulating InP (100) substrate using Au particles with diameter of $30 \mathrm{~nm}$. The samples were annealed under $\mathrm{PH}_{3}$ flow for $10 \mathrm{~min}$ at $450{ }^{\circ} \mathrm{C}$ before carrying out growth at the temperature and V/III ratio of $450{ }^{\circ} \mathrm{C}$ and 350 , respectively. The growth was carried out for $60 \mathrm{~min}$ and the corresponding TMIn and $\mathrm{PH}_{3}$ flows used were $2.0 \times 10^{-6}$ and $7.0 \times 10^{-4} \mathrm{~mol} / \mathrm{min}$.

\section{GaAs WZ NWs}

$\mathrm{In}_{x} \mathrm{Ga}_{1-x} \mathrm{As} / \mathrm{GaAs}$ core/shell NWs were grown by Au-seeded molecular beam epitaxy (MBE) on a deoxidized GaAs (111)B substrate according to the VLS mechanism. Growth temperature was 500

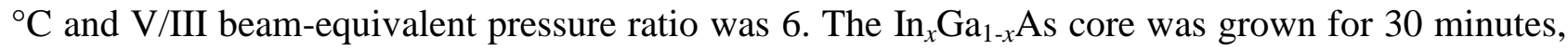


after which the In shutter was closed and GaAs parts (shell and tip) were grown for further 30 minutes. This resulted in a pure WZ GaAs tip elongating for $\sim 800 \mathrm{~nm}$ on top of the core/shell structure. The NWs are vertical on the substrate, $\sim 3 \mu \mathrm{m}$-long, and have a hexagonal cross-section with constant width of $\sim 120 \mathrm{~nm}$ decreasing to $\sim 30 \mathrm{~nm}$ only close to the very end of the NWs. These NWs exhibit a WZ structure with occasional, short ZB inserts.

Table S1 Main characteristics of the investigated samples. Numbers give the NW diameters in nm, as estimated by SEM measurements, except for epilayers, whose thickness is quoted, instead. Crystal phase, growth method, and chemical composition are reported for each diameter value. The fifth column refers to the different types of NWs displayed in Figure 1 in the main text. The last column reports the symbol employed in Figure 4 to individuate samples belonging to different systems.

\begin{tabular}{|c|c|c|c|c|c|}
\hline $\begin{array}{c}\text { NW } \\
\text { diameter }\end{array}$ & $\begin{array}{l}\text { crystal } \\
\text { phase }\end{array}$ & $\begin{array}{l}\text { growth } \\
\text { method }\end{array}$ & $\begin{array}{c}\text { chemical } \\
\text { composition }\end{array}$ & Figure 1 & Figure 4 \\
\hline 61 & $\mathrm{WZ}$ & SAE & InP & (a) & \\
\hline 74 & WZ & VLS & InP & (c) & \\
\hline 108 & WZ & SAE & InP & (a) & \\
\hline 125 & $\mathrm{WZ}$ & SAE & InP & (a) & \\
\hline 130 & WZ & VLS & InP & (b) & \\
\hline 229 & $\mathrm{WZ}$ & VLS & InP & (b) & \\
\hline 335 & WZ & SAE & InP & (a) & \\
\hline 700 & WZ & SAE & InP & (a) & \\
\hline 72 & ZB & VLS & InP & (d) & \\
\hline 139 & $\mathrm{ZB}$ & VLS & InP & (c) & \\
\hline 3000 & $\mathrm{ZB}$ & MOVPE & InP & epilayer & \\
\hline 110 & $\mathrm{WZ}$ & VLS & GaAs & Refs 36 and 37 & \\
\hline 800 & $\mathrm{ZB}$ & MBE & GaAs & epilayer & 交 \\
\hline
\end{tabular}

\section{Bulk samples}

We also investigated a $3.0 \mu \mathrm{m}$-thick InP (100) epilayer grown by MOVPE at $650{ }^{\circ} \mathrm{C}$ and a 0.8 $\mu$ m-thick GaAs (100) epilayer capped by a $1.1 \mu$ m-thick $\mathrm{Al}_{0.17} \mathrm{Ga}_{0.83}$ As layer grown by $\mathrm{MBE}$ at 590 ${ }^{\circ} \mathrm{C}$. Both samples have excellent structural and optical quality. 
Table S1 summarizes the most salient characteristics of the thirteen investigated samples, NWs and epilayers.

\section{S2) Deconvolution of the PL spectra shown in Figure 2 (a) and (b)}

Figures 2 (a) and (b) in the main text show PL spectra recorded at lattice temperatures $\left(T_{\mathrm{L}}\right)$ ranging from 90 to $310 \mathrm{~K}$ on NWs singled out from arrays of WZ InP NWs similar to those displayed in Figure 1 (a) in the main text. The effective diameter, $d$, of the NW in Figure 2 (a) is $d=125 \mathrm{~nm}$ and of the NW in Figure 2 (b) is $d=700 \mathrm{~nm}$. In Figure S2, we show an example of the deconvolution procedure resulting in the fits (dashed black lines) superimposed to the spectra (thick colored lines) in Figure 2. Like in Figure 3 in the main text, we show the spectra recorded at $T_{\mathrm{L}}=310 \mathrm{~K}$. The fits were performed according to Eq. (2) in the main text. Here, we provide the explicit expression of the absorption coefficient ${ }^{1,2}$

$$
\begin{aligned}
\alpha(\hbar \omega)= & \frac{4 \pi e^{2}}{\sqrt{\varepsilon} c \omega m_{0}^{2}}\left|M_{\mathrm{vc}}^{i}\right|^{2}\left\{\frac{1}{r_{\mathrm{exc}, i}^{3}}\left[\sum_{n \geq 1} \frac{1}{n^{3}} A_{\mathrm{b}}\left(E_{\mathrm{g}, i}-\frac{R_{i}}{n^{2}}-\hbar \omega\right)\right]+\right. \\
& \left.+\left(\frac{2 \mu_{\mathrm{exc}, i}}{\hbar^{2}}\right)^{\frac{3}{2}} \frac{\sqrt{R_{i}}}{2} \int_{E_{\mathrm{g}, i}}^{\infty} \frac{A_{\mathrm{b}}(E-\hbar \omega)}{1-\exp \left[-2 \pi \sqrt{R_{i} /\left(E-E_{\mathrm{g}, \mathrm{i}}\right)}\right]} \mathrm{d} E\right\},
\end{aligned}
$$

where $\varepsilon$ is the dielectric constant of the material, $r_{\mathrm{exc}, i}$ is the exciton radius, $\mu_{\mathrm{exc}, i}$ the exciton reduced effective mass, $R_{i}$ the exciton binding energy, and $M_{\mathrm{vc}}^{i}$ the matrix element of the transition considered. $E_{\mathrm{g}, i}-R_{i} / n^{2}=E_{n}^{i}(n=1,2,3, \ldots)$ is the energy of the $n$-th state of the free exciton. In WZ samples, label $i$ ranges from A to $\mathrm{C}$ in order to take into account the contributions from the interband transitions $\mathrm{A}, \mathrm{B}$, and $\mathrm{C}$ with energy gap $E_{\mathrm{g}, i}$. These three contributions were summed up. In ZB samples, we have considered only the band gap transition and the $i$ label is omitted. The terms within square brackets in the first row take into account the exciton states. The addend in the second row takes into account the absorption due to band-to-band transitions; therein, the exponential function in the integral accounts for the Sommerfeld factor due to the Coulomb 
interaction between unbound electrons and holes. $A_{\mathrm{b}}$ is a suitable broadening function (Gaussian or Lorentzian).

In Figure $\mathrm{S} 2$, exciton $\left(\mathrm{E}_{1}\right.$ is the energy of the $n=1$ exciton ground state) and band-to-band $\left(\mathrm{E}_{\mathrm{b}-\mathrm{b}}\right)$ contributions are represented by blue and orange filled areas, respectively. Values of the parameters entering into $\alpha(\hbar \omega)$ for a certain optical transition (dielectric constant, exciton radius and reduced effective mass), as well as the broadening functions, are the same as those in Ref. 2. Fitting parameters are the relative amplitude of the different contributions, the band-gap energies, the fullwidth at half-maximum of the broadening functions, and the carrier temperatures. A same carrier temperature $\left(T_{\mathrm{C}}\right)$ was used for $\mathrm{A}, \mathrm{B}$, and $\mathrm{C}$ transitions. There is a very good agreement between experimental data and fits, with the small disagreement on the low-energy side of the spectra being due to the presence of defect states not included in the fitting procedure. $T_{\mathrm{C}}$ and $\Delta T=T_{\mathrm{C}}-T_{\mathrm{L}}$ values, obtained by the fits and reported also in Figure 2 (c) in the main text, are provided.
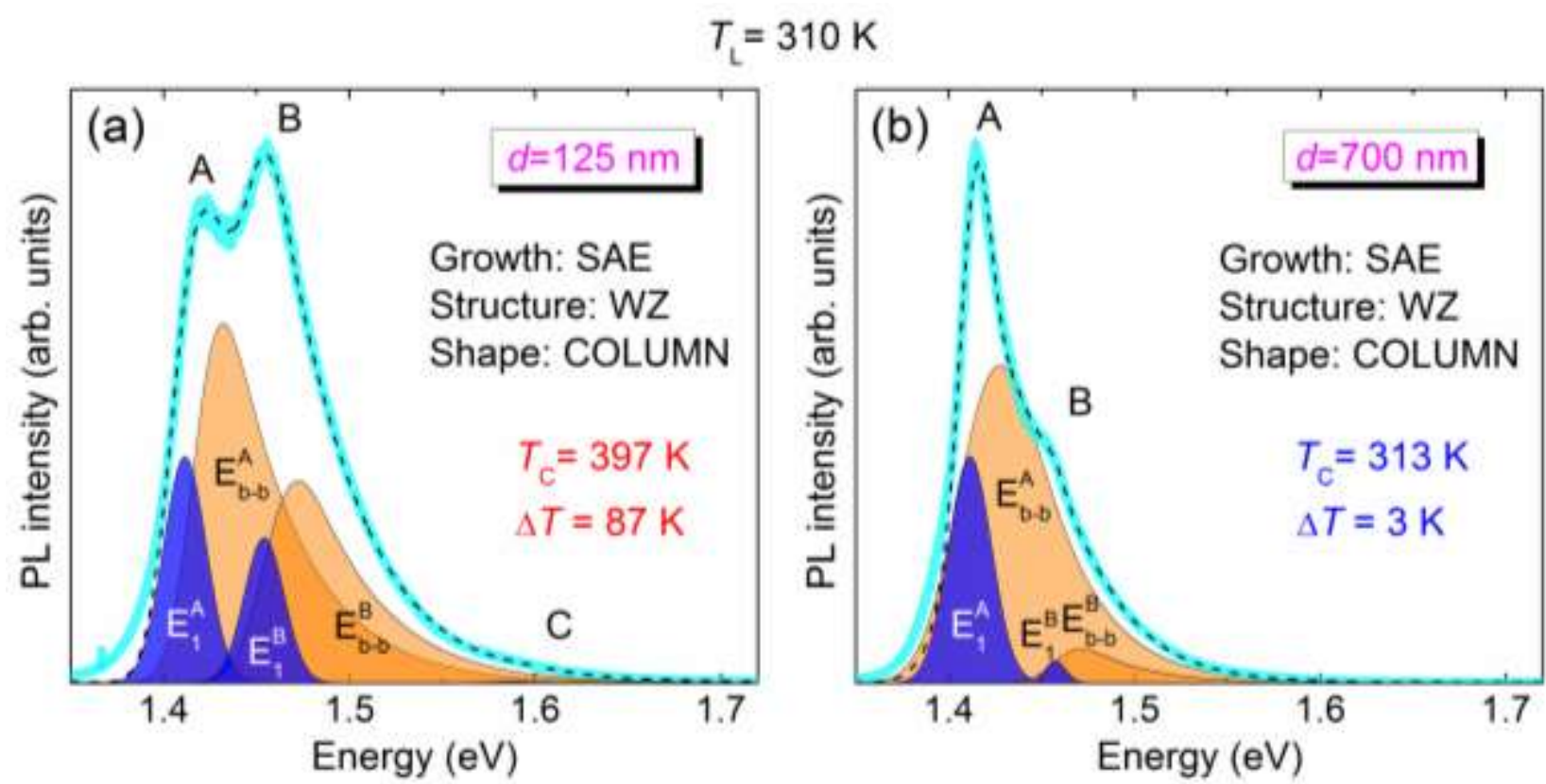

Figure S2 PL spectra (thick colored lines) recorded at $T_{\mathrm{L}}=310 \mathrm{~K}$ on single WZ InP NWs grown by SAE and already shown in Figure 2 in the main text. The spectrum in (a) was recorded on a NW with $d=125 \mathrm{~nm}$ at power density $P=1.14 \mathrm{~kW} / \mathrm{cm}^{2}$; the spectrum in (b) was recorded on a NW with $d=700 \mathrm{~nm}$ at $P=0.51$ $\mathrm{kW} / \mathrm{cm}^{2}$. Dashed black lines are best fits to the data via Eq. 2 in the main text. The various contributions are indicated by blue-filled areas for excitons (labeled as $\mathrm{E}_{n}$, with $n=1$ for the ground state) and by orange-filled areas for continuum states (labeled as $\mathrm{E}_{\mathrm{b}-\mathrm{b}}$ ). The superscripts $\mathrm{A}, \mathrm{B}$, and $\mathrm{C}$ associate the contributions to the different transitions. For clarity purposes, in (a) small contributions $\mathrm{E}_{1}^{\mathrm{C}}$ and $\mathrm{E}_{\mathrm{b}-\mathrm{b}}^{\mathrm{C}}$ from transition $\mathrm{C}$ have not been shown, although they have been included in the fit. In (b) transition $\mathrm{C}$ is not observable. 
Figure $\mathrm{S} 2 \mathrm{~b}$ shows on a semilog scale the PL spectrum (thick red line) recorded at $T_{\mathrm{L}}=290 \mathrm{~K}$ on an ensemble of ZB InP NWs, whose SEM is shown in Figure 1 (d) of the main text. The dashed line is a fit to the data via Eq. (2) in the main text. The carrier temperature determined by the fit is $T_{\mathrm{C}}^{\mathrm{fit}}=397 \mathrm{~K}$. Instead, the value of $T_{\mathrm{C}}$ determined directly by the slope of $\ln \left[I_{\mathrm{PL}}(\hbar \omega)\right] v s \hbar \omega$ is $T_{\mathrm{C}}^{\text {slope }}=449 \mathrm{~K}$, clearly overestimating the actual carrier temperature. Therefore, a proper fitting routine taking into account the energy dependence of the absorption coefficient too must be employed to derive the correct $T_{\mathrm{C}}$ values.

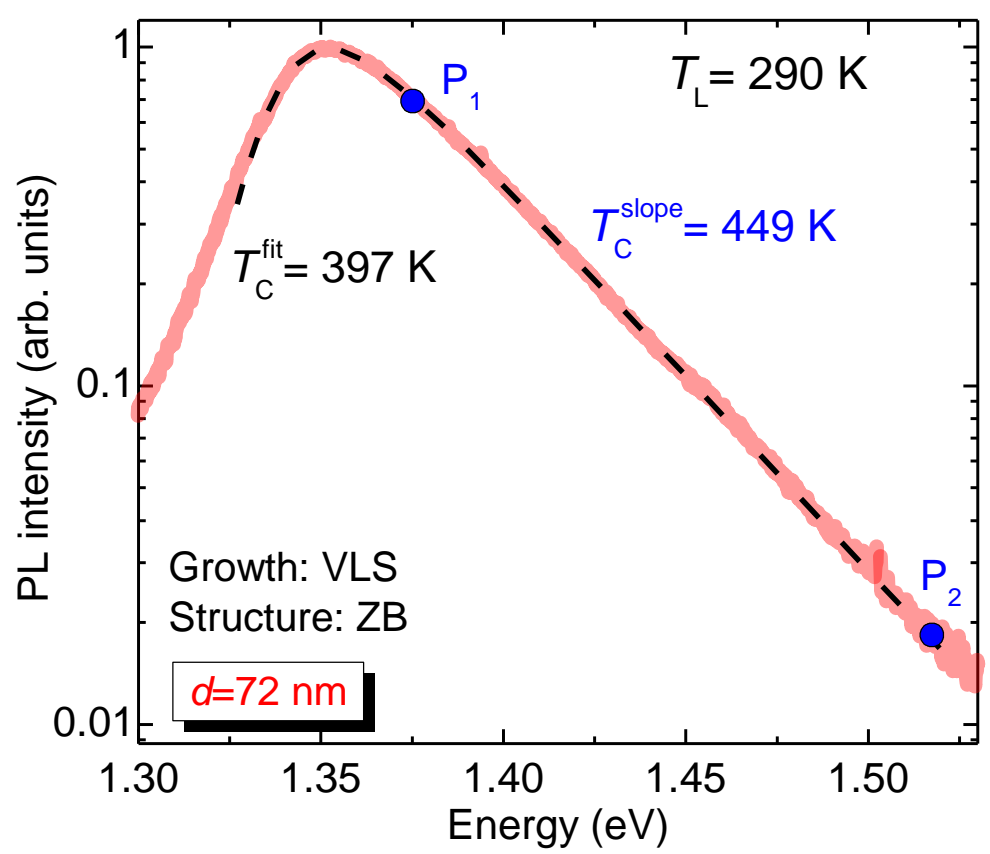

Figure S2 b PL spectrum (thick red line) recorded at $T_{\mathrm{L}}=290 \mathrm{~K}$ and power density $P=6.4 \mathrm{~W} / \mathrm{cm}^{2}$ on an ensemble of ZB InP NWs grown by VLS. The NW average ensemble diameter is $d=72 \mathrm{~nm}$. The dashed black line is a best fit to the data via Eq. 2 in the main text resulting in a carrier temperature $T_{\mathrm{C}}^{\mathrm{fit}}=397 \mathrm{~K}$. $\mathrm{P}_{1}$ and $\mathrm{P}_{2}$ indicate two data points from which we extract the carrier temperature using the slope of $\ln \left[I_{\mathrm{PL}}(\hbar \omega)\right] v s \hbar \omega$ resulting in a sizably larger carrier temperature, $T_{\mathrm{C}}^{\text {slope }}=449 \mathrm{~K}$.

\section{S3) PL spectra and fits of all samples at lattice temperature of $290 \mathrm{~K}$}

Figure 4 in the main text shows the difference between carrier and lattice temperatures $\Delta T=T_{\mathrm{C}}-T_{\mathrm{L}}$, as a function of the effective diameter of the samples for $T_{\mathrm{L}}=290 \mathrm{~K}$. Carrier temperatures were extracted by the PL spectra recorded on all the InP and GaAs samples listed in the Samples and 
Experimental Details section in the main text. All these spectra are shown in Figure S3 by thick colored lines, along with fits performed using Eq. 2 (black dashed lines) that enabled the determination of $T_{\mathrm{C}}$ and thus $\Delta T$.
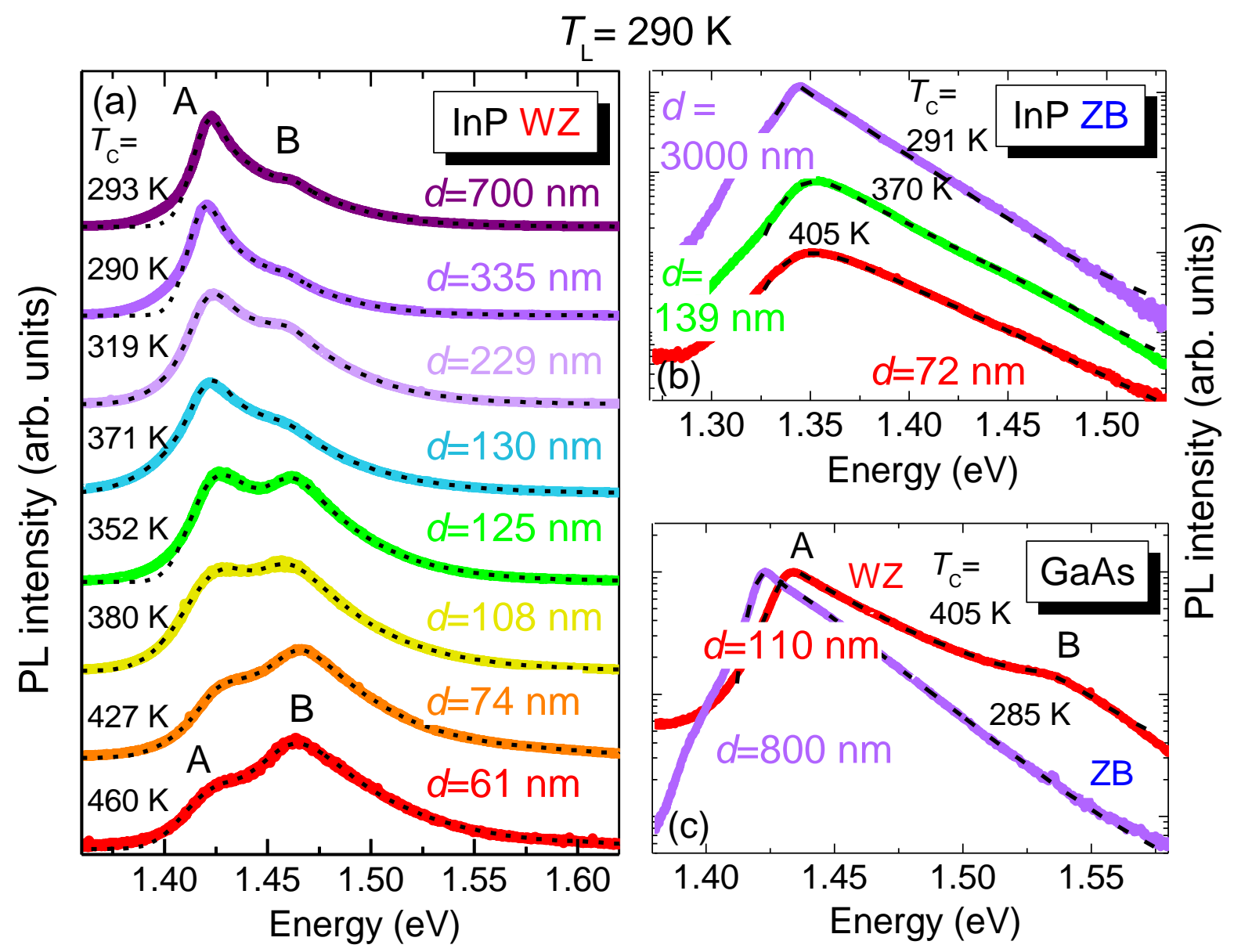

Figure S3 PL spectra (thick colored lines) recorded at $T_{\mathrm{L}}=290 \mathrm{~K}$ on all the samples of Figure 4 in the main text. The carrier temperature $T_{\mathrm{C}}$ as determined by fits is provided for each sample. Panel (a) refers to WZ InP NWs, (b) to ZB InP NWs and epilayer, and (c) to WZ GaAs NWs and ZB epilayer. In WZ samples, A and B bands are indicated. The diameter $d$ labeling each spectrum allows finding samples properties as listed in Table 1 and the inset of Figure 4. Dashed black lines are fits to the data using Eq. 2, whose resulting $\Delta T$ are provided in Figure 4. The small mismatch between data and fits on the low-energy side of the spectra is due to contributions from defect states not considered in the fittings. Notice the semilog scale in panels (b) and (c).

Spectra were recorded in the experimental configurations summarized in the description of Figure 4 in the main text, and sample characteristics are provided in Table S1. Here, panel (a) refers to InP NWs with WZ structure, panel (b) to InP samples with ZB structure (the sample with $d=3000$ $\mathrm{nm}$ is the epilayer), and panel (c) to GaAs samples (WZ NWs with $d=110 \mathrm{~nm}$ and the $800 \mathrm{~nm}$-thick 
ZB epilayer). Notice the semilog scale in (b) and (c). In WZ samples, A and B bands are indicated. In WZ GaAs, we find band B about $110 \mathrm{meV}$ above band A, in agreement with Refs. 3 and 4.

Carrier temperature increases with decreasing the effective diameter of the NWs. In panels (b) and (c), at first glance such an increase appears on the high-energy tail of the spectra due to the increase in the slope. In panel (a), we notice that the smaller the diameter, the higher band B population, because carriers gain a larger thermal budget $\Delta T=T_{\mathrm{C}}-T_{\mathrm{L}}$ in NWs with smaller $d$. In turn, this enables a larger thermal population of high-energy states. This applies also to WZ GaAs NWs; see panel (c). Therein, however, band B is less populated than in InP NWs with a similar diameter (thus with a similar thermal budget), because the energy difference between A and B is much larger in WZ GaAs (110 meV) than in WZ InP (40 meV).

\section{S4) Power-dependent PL studies}

In order to investigate the dependence of carrier temperature on power density in samples that exhibit as well as that do not exhibit the hot carrier phenomenon, PL spectra were recorded at variable power densities on four representative InP NW samples with different diameters and crystal structure, as shown in Figure S4.

Spectra in panel (a) were taken at $T_{\mathrm{L}}=310 \mathrm{~K}$ on an ensemble of WZ NWs with $d=130 \mathrm{~nm}$; its SEM image is displayed in Figure 1 (b) in the main text. Spectra in (b), taken at $T_{\mathrm{L}}=290 \mathrm{~K}$, were recorded on WZ NWs with $d=700 \mathrm{~nm}$ similar to the NWs whose SEM is shown in Figure 1 (a) in the main text. In both samples, the spectra were recorded with a macro-PL set-up. Micro-PL was employed, instead, to record the spectra shown in panels (c) and (d), referring respectively to a single WZ and a single ZB InP NW transferred on a Si substrate. The WZ NW was singled out from the WZ section of a kinked NW belonging to the ensemble whose SEM picture is displayed in Figure 1 (c) of the main text. The ZB NW was singled out from the ZB section of another kinked NW. In all cases, the PL line shapes do not change sizably with laser power density, $P$. 

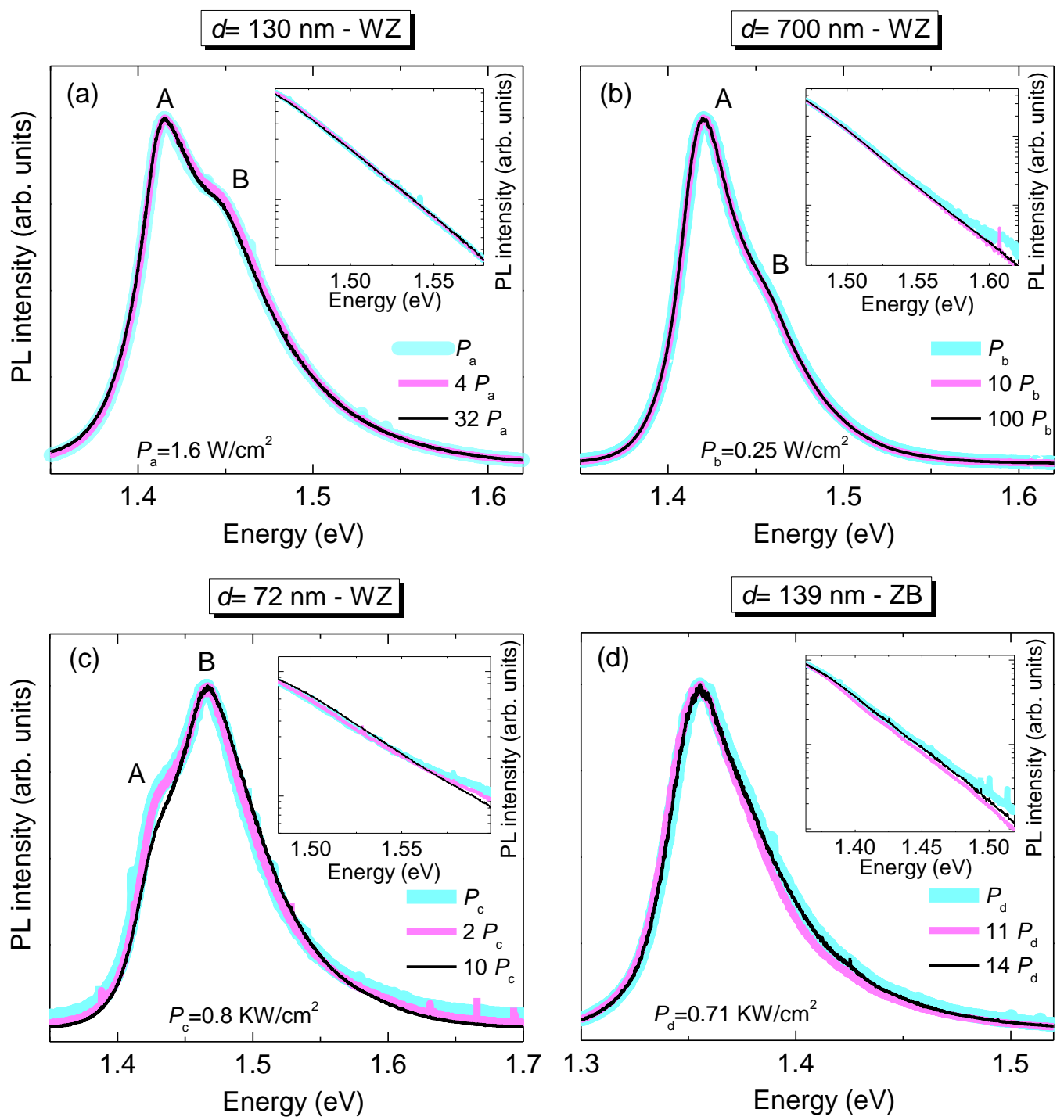

Figure S4 Power-dependent PL spectra at room temperature $\left(T_{\mathrm{L}}=290-310 \mathrm{~K}\right.$, depending on sample) for NWs with different diameters, crystal phase, and experimental configuration. (a) and (b) refer to macro-PL measurements on ensembles of WZ InP NWs of different diameters. (c) and (d) refer to micro-PL measurements performed on a single InP NW with different diameter and different crystal phase [WZ and ZB for (c) and (d), respectively]. Spectra were taken at three power densities, as detailed in each panel. Notice the nearly complete overlap of the PL line shape for different excitation power densities, except for in panel (c). In any case, the carrier temperature does not change with laser power density, as highlighted by the insets in each panel that show the high-energy tail of the spectra on a semilog scale.

In particular, the carrier temperature remains constant with increasing $P$. This is highlighted in the insets that show that the high-energy tail of the PL spectra does not vary much with laser power density. Seemingly different carrier temperatures due to slightly varying slopes in the insets are caused by background contributions [see e.g. the different baselines on the low energy side in panel (c)]. In any event, lineshape analysis ensures that carrier temperature does not change sizably with 
$P$. It should be also noticed that the energies of $\mathrm{A}, \mathrm{B}$, and $\mathrm{C}$ peaks do not change with power variation. Owing to the well-known band-gap thermal shrinkage, this indicates a constant lattice temperature at all powers and allows us to rule out any heating effect of the lattice induced by laser excitation.

We point out that the higher population of B band in (c) than in (a) and (b) [panel (d) refers to a ZB NW], confirms a higher $T_{\mathrm{C}}$ in the NW with the smallest diameter despite the similar $T_{\mathrm{L}}$ of the diverse samples.

\section{S5) High-resolution TEM images of the nanowire surfaces}

Figure S5 displays high-resolution transmission electron microscopy (HR-TEM) images of some representative InP NWs showing the degree of surface roughness. Following the same sequence used in Figure 1 in the main text, here panel (a) refers to a WZ NW grown by seed-free SAE, panel (b) to a highly tapered WZ NW, panel (c) to a kinked NW, whose WZ section is displayed on the left and the ZB section on the right, and panel (d) to a slightly tapered ZB NW. The effective diameter of each NW is provided in the figure caption. Clearly, all NW surfaces are rather smooth, that makes the role played by surface roughness negligible in determining the dependence of hot carrier temperature on NW diameter. 

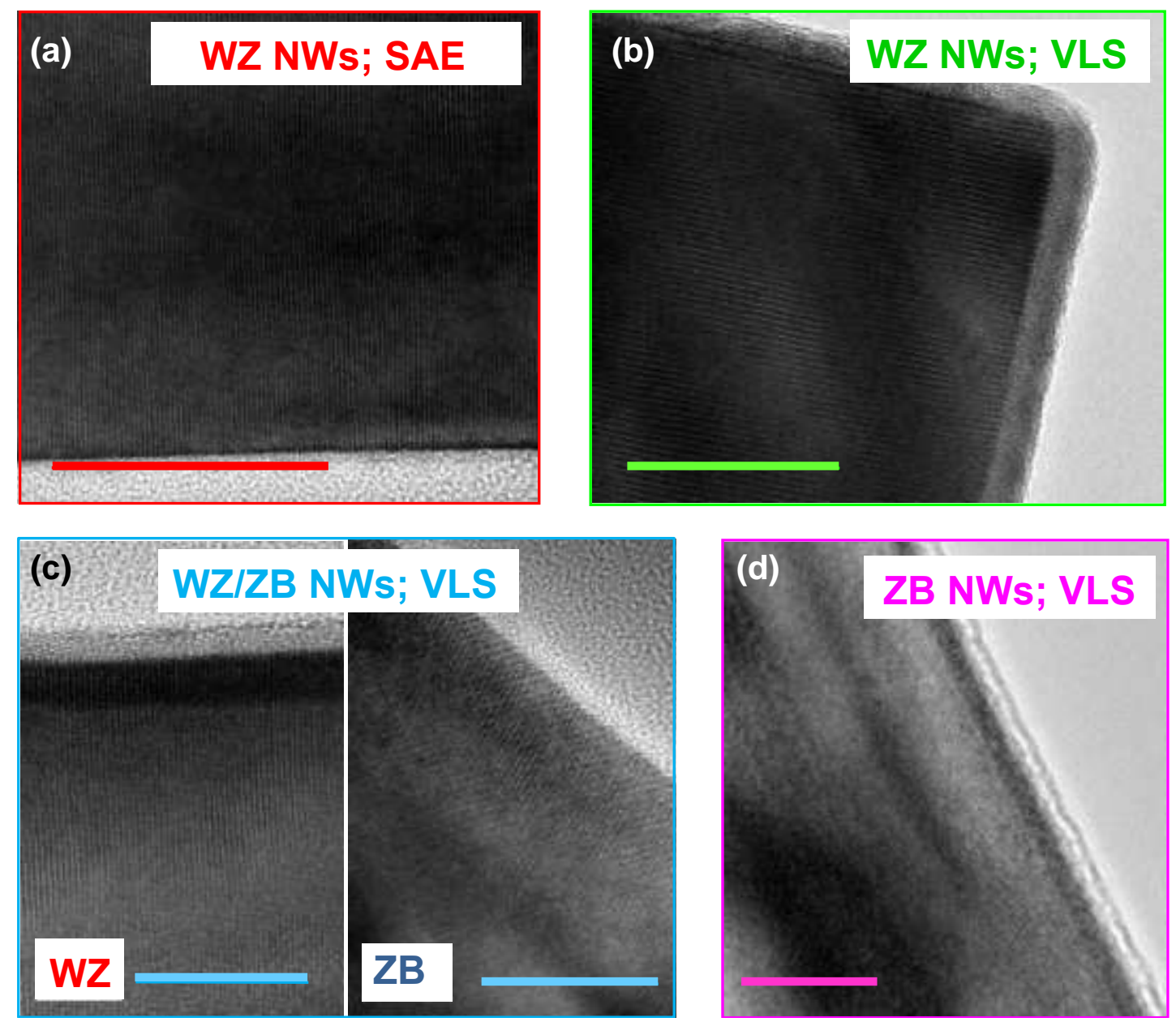

Figure S5 HR-TEM images of the surface of the InP NWs whose SEM images are shown in Figure 1 in the main text. Scale bars are $20 \mathrm{~nm}$. (a) Taper-free NW grown by metal-free SAE and having pure WZ structure $(d=130 \mathrm{~nm})$. (b) Highly tapered WZ NW grown by Au-seeded MOVPE $(d=229 \mathrm{~nm})$. (c) Taper-free NW grown by Au-seeded MOVPE. The left image is of a WZ segment $(d=74 \mathrm{~nm})$, the right is of a ZB segment $(d=139 \mathrm{~nm})$. (d) Slightly tapered ZB NW grown by Au-seeded MOVPE $(d=72 \mathrm{~nm})$. Notice that the fringe visible in light contrast on the surface of the NW is due to damage induced by the electron beam.

\section{REFERENCES}

[1] Grilli, E.; Guzzi, M.; Zamboni, R.; Pavesi, L. Phys. Rev. B 1992, 45, 1638-1644.

[2] Zilli, A.; De Luca, M.; Tedeschi, D.; Fonseka, H. A.; Miriametro, A.; Tan, H. H.; Jagadish, C.; Capizzi, M; Polimeni, A. ACS Nano 2015, 9, 4277-4287.

[3] De Luca, M.; Lavenuta, G.; Polimeni, A.; Rubini, S.; Grillo, V.; Mura, F.; Miriametro, A.; Capizzi, M.; Martelli, F. Phys. Rev. B 2013, 87, 235304 1-8.

[4] Ketterer, B.; Heiss, M.; Uccelli, E.; Arbiol, J.; Fontcuberta i Morral, A. ACS Nano 2011, 5, 7585-7592. 